\title{
Decay studies of heavy and superheavy nuclei
}

\author{
Roderick M. Clark ${ }^{\mathrm{a}}$ \\ Nuclear Science Division, Lawrence Berkeley National Laboratory, Berkeley, CA 94720, USA
}

\begin{abstract}
In this contribution I discuss recent results on the decay of excited states, via electromagnetic transitions ( $\gamma$-ray and conversion electrons), $\alpha$ decay, and fission, and discuss what these studies reveal in terms of the stability of the heaviest elements.
\end{abstract}

\section{Introduction}

What is the heaviest element? This seemingly simple question still evades an answer. Determining $\gamma$ which combinations of protons and neutrons can form a nucleus stable enough to acquire electrons and form a neutral atom (a process with a characteristic time scale on the order of femtoseconds) is one part of the solution, but the nucleus must also survive long enough to be observed. Typical experimental set-ups of today, using large-acceptance separators, require that the nucleus survive around a microsecond for there to be a chance of observation.

Since superheavy elements owe their existence to the additional stability that arises from gaps in the quantal shell structure of the nucleon levels, it is particularly important to learn about the single-particle states of the heaviest nuclei. There has been a great deal of recent work involving both prompt and delayed $\gamma$-ray spectroscopy, measuring the properties of excited states in nuclei from Fm $(Z=100)$ on up [1,2]. One can learn not only about the single-particle structure but also about the role of pairing, and the different shapes and excitation modes of the heaviest nuclei. These fundamental properties can then be used to test the current models, the same models that are used to make predictions of the extent of the heaviest elements.

While the single-particle structure is vital to understanding the stability of the heaviest elements, the $\alpha$ decay and fission processes ultimately determine how long a nucleus will survive. Recent observations in the decay chains of ${ }^{270}$ Ds [3, 4] suggest that high- $K$ isomeric states can decay via $\alpha$ emission where the metastable state is longer lived than the ground state of the same nucleus. Moreover, recent results on ${ }^{254} \mathrm{Rf}[5]$ suggest that such high- $K$ states can also have a significant hindrance against fission. Such results have tremendous implications for how far we may be able to push the studies of the heaviest elements and it is important to gain a better understanding of these decay mechanisms.

In this contribution I describe recent results from work done at Lawrence Berkeley National Laboratory, which address the issues of single-particle structure of the heaviest nuclei, and the $\alpha$-decay and fission hindrances of $K$-isomers. First, I briefly discuss the latest $\gamma$-ray spectroscopy of the decay chains of ${ }^{288} 115$ [6-8], and the implications that such studies have on the knowledge of single-particle states. This leads on to a more general discussion of the predictions of theoretical models, especially concerning the occurrence and extent of

\footnotetext{
a e-mail: rmclark@lbl.gov
} 


\section{Comparison of Theories}

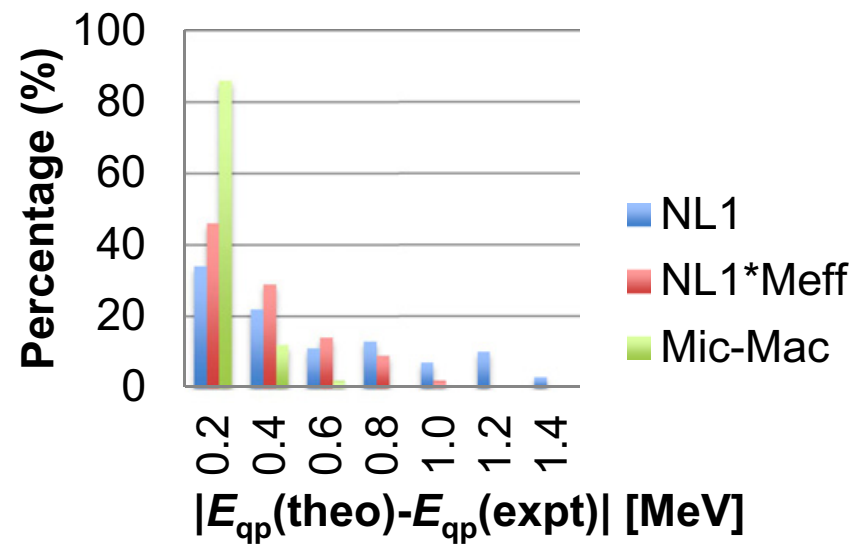

Figure 1. Distribution of the deviations of the calculated energies, $E_{\mathrm{qp}}$ (theo), of one-quasiparticle states from the experimental ones, $E_{\mathrm{qp}}$ (expt). The vertical axis shows the percentage of the states that deviate from experiment by the energy range specified on horizontal axis. NL1 refers to a particular interaction used in the microscopic calculation [20], while NL1*Meff indicates an ad hoc compression factor (see text). The Mic-Mac model refers to the calculations described in reference [18, 19].

spherical and deformed shell structure. Next, I describe efforts to understand the stability of metastable states, notably $K$-isomers in regions near the deformed shell gaps, against both $\alpha$ decay and fission. In the case of $\alpha$ decay, I describe the use of a simple superfluid-tunneling model to understand the role of pairing in enhancing the stability of the metastable states in superheavy nuclei, and use a hypothetical case in ${ }^{294} 118$ as an example [9]. For the fission of these states I attempt to answer the very simple question of how much one might expect the fission hindrance of a two-quasiparticle $K$-isomer to change relative to the corresponding ground state of an even-even nucleus.

\section{Gamma-decay spectroscopy and single-particle structure}

It is extraordinary to think that we are now able to perform $\gamma$-ray spectroscopy on nuclei in the decay chains of super-heavy isotopes. Two experiments were performed recently, one at GSI and a second at LBNL, using the ${ }^{48} \mathrm{Ca}+{ }^{243} \mathrm{Am}$ reaction to create isotopes of element115 , notably ${ }^{288} 115$ via the three-neutron evaporation channel. Combining the results from the two experiments, more than seventy chains of $\alpha$ decays were observed and assigned to the decay of this isotope $[6-8,10]$. It was already known that there was a fine structure observed in the $\alpha$ decay [11] indicative of it passing through both the ground states and excited states in daughter isotopes belonging to these chains. It was hoped that the excited states might involve a competitive electromagnetic decay via some highly converted $\gamma$-ray transition, which would result in the observation of $X$-rays, confirming the assignment of the atomic number.

No clear-cut $X$-ray spectrum was observed, but multiple $\alpha$-photon coincidences were seen, and discrete $\gamma$-ray transitions in the decay schemes of ${ }^{276} \mathrm{Mt}$ and ${ }^{272} \mathrm{Bh}$ were identified (see Fig. 1 of Ref. [7] and Fig. 2 of Ref. [8] to see the spectra and their interpretations). The fact that there is no apparent $X$-ray spectrum observed is important since it indicates that many transitions identified in these nuclei are not highly converted and most likely have electric dipole character. In turn, this indicates that the initial and final states involved in the decay have opposite parity. This is sufficient to place constraints on the models of 


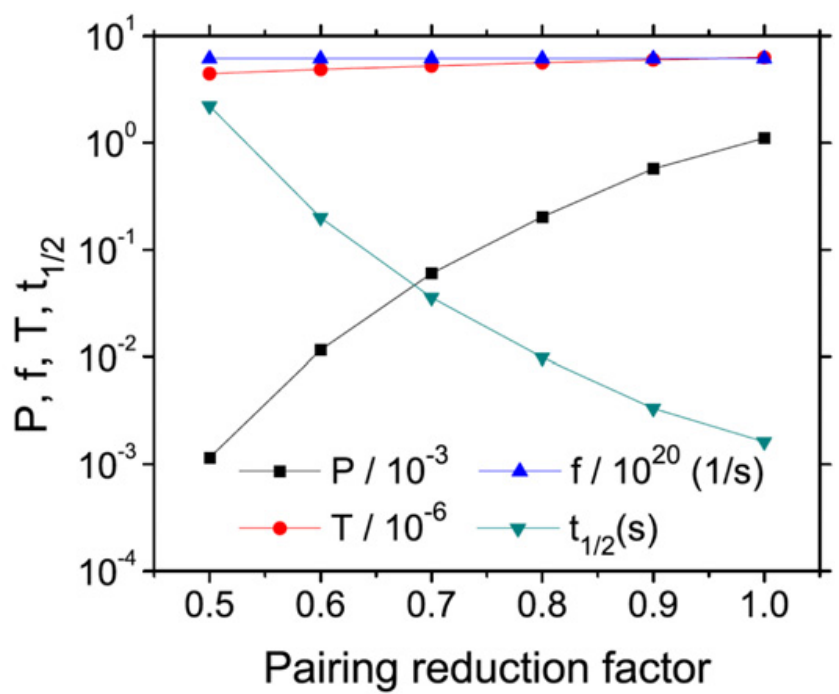

Figure 2. Contribution of the pairing reduction on the different factors $f, P$, and $T$, needed to calculate the half life, $t_{1 / 2}$, on the $\alpha$ decay of a hypothetical two-quasiparticle isomeric state in ${ }^{294} 118$. See text for more details.

single-particle structure for these nuclei [12]. These studies of decay chains of superheavy elements are extreme examples of $\gamma$-ray spectroscopy (involving cross-sections of only a few picobarns) and represent the first tentative steps toward more detailed studies, which should provide the most stringent tests of theory.

There are two broad categories of theory, which are making predictions of the single-particle structure of the heaviest elements. The first category involves microscopicmacroscopic (mic-mac) models [13-15]. These models involve describing the bulk properties of the nuclei through macroscopic approaches (such as the liquid drop model) and then superposing the microscopic (quantum) level structure. These approaches are often "tuned" to give the best reproduction of available experimental data and the issue is often raised of how well this category of model can be extrapolated to make predictions of the heaviest elements. The second category of models involves self-consistent microscopic approaches [20]; in today's parlance density functional theories (DFT) using effective nucleon-nucleon interactions. The interactions are usually fitted to the bulk properties of a few key nuclei (e.g., near ${ }^{48} \mathrm{Ca}$ and ${ }^{208} \mathrm{~Pb}$ ) rather than being fitted to local level structure, while the self-consistency leads to hopes that these models may be more reliably extrapolated to new regions such as the superheavy nuclei.

While these two approaches represent rather different philosophies, it is clear that any successful theory must be able to reproduce the available experimental data. Most microscopic-macroscopic treatments predict that there are major spherical shells at $Z=114$ and $N=172$, while the DFT approaches predict that the gaps could occur at $Z=114$, $Z=120$, or even $Z=126$ and with neutron numbers $N=172$ or $N=184$. However, both categories of theory predict regions of low orbital degeneracy near these magic numbers, corresponding to extended regions of substantial shell stabilization, and the current experimental data is unable to differentiate between the models on this basis alone.

However, the differences between the theories should also be highlighted by how well they reproduce the known level structure of nuclei in the actinide and trans-actinide region, where there is more detailed experimental information. In Fig. 1, we show a comparison 
between theory and experiment. We have used the available experimental data on quasiparticle energies (differing from single-particle energies since the measured level energy determined from experiment is not a pure single-particle state and the effect of pairing must be taken into account) twenty-one odd- $N$ and twenty-three odd- $Z$ nuclei in the region with $Z=89$ to $Z=100$. The experimental data is compared against two models representative of microscopic-macroscopic [18] and the fully microscopic [20] approaches, respectively.

One clearly sees that the microscopic approach can only reproduce about one-third of the experimental level energies to within $200 \mathrm{keV}$ and differences of around $1 \mathrm{MeV}$, or more, are quite common. Even after introducing an ad-hoc term, which "compresses" the theoretical level structure, less than half the experimental level energies are reproduced to within $200 \mathrm{keV}$, indicating that the level ordering is often incorrectly predicted. By contrast, the microscopic-macroscopic model reproduces over $85 \%$ of the known experimental levels to within $200 \mathrm{keV}$. This suggests that, from a purely pragmatic point-of-view, the mic-mac models better reproduce experiment and provide the most useful predictions. While the DFT approaches may be more readily extrapolated, they are not able to reliably reproduce the known experimental information, and their predictions of single-particle structure near the major spherical gaps have limited value in guiding experiment.

\section{Alpha decay of ground and isomeric states}

One consequence of the single-(quasi) particle structure is the possibility of the occurrence of isomers in specific regions of nuclei. Of particular interest are regions of high- $K$ isomerism in deformed superheavy nuclei. These isomers arise because they involve configurations of nucleon orbitals, which yield high- $K$ values, where $K$ is the projection of the total angular momentum onto the axis of symmetry defined by the nuclear shape. Electromagnetic decays from these states may involve an energetically unfavorable re-orientation of nucleons to states with much lower $K$ values and such transitions can become hindered, leading to the metastability. The decay of these high- $K$ isomers have proven to be a useful tool for $\gamma$-ray spectroscopy in nuclei near $Z=100$ and $N=152\left({ }^{252} \mathrm{Fm}\right)[1,2]$. However, it is the recent observation of $\alpha$ decay from high- $K$ isomers in ${ }^{270} \mathrm{Ds}(Z=110)$ and in the daughter ${ }^{266} \mathrm{Hs}$ that is of interest here [3,4]. Not only do these isomers $\alpha$ decay, but the lifetimes of the isomeric states are longer than the corresponding ground state. This has profound implications for the survival of superheavy nuclei and the possibility of studying these states experimentally.

There are three major factors which influence the $\alpha$ decay of multi-quasiparticle states: i) the energies of the states involved - the larger the $Q$-value of the $\alpha$ decay the shorter the lifetime will be; ii) a large difference in angular momentum will give rise to a larger centrifugal barrier resulting in a longer lifetimes; iii) pairing enhances the decay through a barrier and so a reduction in pairing again implies a longer lifetime. Determining the relative influences of these competing effects is important for our understanding of the $\alpha$ decay of the excited states and the survival probability for superheavy elements.

In order to address this issue we have used the superfluid-tunneling model [21-23] to estimate these effects and apply it to the case of a superheavy nucleus [9]. The decay constant, $\lambda$, may be expressed as $\lambda=P \times f \times T$, where $P$ is the preformation factor of the $\alpha$ particle, $f$ is the frequency of the $\alpha$ particle hitting the barrier (the "knocking" frequency), and $T$ is the transmission coefficient through the barrier. The methodology for calculating each term is explained in detail in [24]. We first applied the model to known ground-state-to-groundstate $\alpha$ decays and it reproduced the experimental data very well. For example, the calculated half-lives of the decays were found to be within a factor of 2.5 of the experimental values, comparable to the best semi-empirical models (see Fig. 1 of Ref. [9]). Next we used the 
same approach to try to reproduce the lifetimes for the $\alpha$ decays of multi-QP isomers in the neutron-deficient rare-earth and light actinide regions. By including a common pairing reduction factor of 0.6 we are able to reproduce the experimental data to within a factor of 3.5 (see Fig. 4 of Ref. [9]).

The ability to reproduce the known experimental data quite accurately gives confidence that the model contains the physical ingredients that are essential to understanding the decay process. We then turned to calculating a hypothetical case of relevance to the superheavy nuclei: we assume a two-quasiparticle $K^{\pi}=10^{+}$isomeric state at $E=1.05 \mathrm{MeV}$ in the nucleus ${ }^{294} 118$. By modifying the pairing gap we calculated the effect on factors $P, f, T$, and $t_{1 / 2}$, as shown in Fig. 2. To form an $\alpha$ particle, a pair of neutrons, and a pair of protons, need to be coupled together. Therefore, the reduction in the pairing interaction has a remarkable effect on the $\alpha$-particle preformation factor. The measured half-life of the ground-state of element ${ }^{294} 118$ is $t_{1 / 2}=0.69 \mathrm{~ms}$, which is one to two orders of magnitude shorter than the theoretical isomeric state when the pairing reduction factor of 0.6 is used as before. Therefore, in regions where a stiff axial deformation persists, we can expect the heaviest elements to have isomeric states with longer half-lives than their ground states.

\section{Fission decay of high-K states}

Recent experiments on high- $K$ isomers in the transfermium region have suggested that these metastable states may also have "unprecedented fission hindrance relative to the ground state" [5]. Since fission is the process that will ultimately dictate the survival of the heaviest elements it is important to understand this claim. A case of topical interest is that of ${ }^{254} \mathrm{Rf}$ [5]. Note, a compilation of all prior known data on $K$-isomer fission hindrances can be found in [25]. The ground state decay of ${ }^{254} \mathrm{Rf}$ is dominated by fission and has a half-life of 23.2(11) $\mu$ s [5]. An exploratory run at Argonne National Laboratory using the Fragment Mass Analyzer (FMA) at the ATLAS accelerator facility, provided evidence for the occurrence of at least one high- $K$ isomer in this nucleus. A second experiment, using the Berkeley Gasfilled Separator at the 88-Inch Cyclotron of Lawrence Berkeley National Laboratory (LBNL) was performed in order to obtain detailed information on the decay of such high- $K$ states. In particular, it seemed reasonable to think that since the ground state decay was dominated by fission, then one might expect that any isomers in this nucleus could also have a significant fission branch. Definitive identification of fission decay of high- $K$ states could provide a new probe of the fission process revealing information about the role of pairing and the influence of $K$ conservation on the process.

The results of the second experiment revealed the presence of two isomers, one feeding the other, which decay by electromagnetic transitions with half-lives of 4.7(11) $\mu$ s and 247(73) $\mu \mathrm{s}$. The isomer with the shorter lifetime was interpreted as a two quasi-neutron state based on the $K^{\pi}=8^{-}\left\{v^{2}\left(7 / 2^{+}[624], 9 / 2^{-}[734]\right)\right\}$ configuration. The longer-lived isomer was interpreted as a four quasi-particle state with the $K^{\pi}=16^{+}\left\{v^{2}\left(7 / 2^{+}[624], 9 / 2^{-}[734]\right) \pi^{2}\left(7 / 2^{-}[514], 9 / 2^{+}[624]\right)\right\}$ configuration. We were able to estimate that the fission partial lifetimes are more than 2 (25) times longer for the two-(four)quasi-particle isomers, respectively. The absence of any sizeable fission branch from either of the isomers was suggested as evidence of the claim of "unprecedented fission hindrance".

While the experimental results are exciting it is unclear what one a priori expects in terms of the fission hindrance of a high- $K$ state. The excitation energy of a high- $K$ two-quasiparticle isomer in this region is at least $1 \mathrm{MeV}$ (roughly two times the pairing gap). In a simplified picture of a single parabolic barrier one could equate this with a lowering of the barrier height by $1 \mathrm{MeV}$. This would result in a shortening of the fission half-life. However, a high- $K$ state 
involves broken pairs and in some sense it "looks" more like the configuration of the ground state of an odd-odd neighbour. One would then expect the half-life for fission to increase due to effects from the odd particles (often loosely described in terms of a "specialization energy" which accounts for the angular momentum, $K$ value, and reduced pairing correlations).

In order to estimate the effect of the higher excitation energy of the high- $K$ state we draw on the analysis of Vandenbosch and Huizenga for a simple parabolic fission barrier [26]. The fission half-life can be expressed as: $t_{1 / 2}=2.77 \times 10^{-21} \exp \left[2 \pi\left(B_{f}\right) / \hbar \omega\right]$. Assuming a constant barrier curvature of $\hbar \omega=0.5 \mathrm{MeV}$ and changing the fission barrier height, $B_{f}$, from $6 \mathrm{MeV}$ to $5 \mathrm{MeV}$ one finds that the isomeric state would decay with a half-life that is $\sim 3 \times 10^{5}$ times faster than the ground state.

To estimate the effect of the "odd-odd character" of the high- $K$ isomer on the fission hindrance we turn to an empirical estimate by looking at the hindrance factors of fission half lives of the ground states of odd- $Z$ and odd- $N$ neighbours of even-even nuclei. It is well known that the hindrance factor [27] arising from the odd particle, whether proton or neutron, is typically of the order of $10^{3}$ to $10^{5}$. There are very few cases where the odd-odd neighbour has a useful value, or limit, placed on the fission hindrance arising from the two odd particles, but it seems reasonable to expect that the effect of each odd particle is to contribute such that the odd-odd character would result in hindrances of $\sim 10^{8}$. One then can suggest that it would be reasonable to expect a fission hindrance for a two quasiparticle high- $K$ state to be about $10^{3}$ relative to the ground state. Limits, such as those established for the high- $K$ isomers in

${ }^{254} \mathrm{Rf}$, are significantly lower than this expectation. Indeed, looking at all the available data on cases where there are claims of fission from high- $K$ isomeric states [25], several of the reports appear suspect. It is likely that experiments must improve their sensitivity by several orders of magnitude if we hope to understand fission from isomeric states and to use such observations as a new probe of the fission process.

\section{Summary}

In this contribution I have looked at some aspects of the electromagnetic, $\alpha$, and fission decay of the heaviest elements. These processes can provide information on the stability of superheavy nuclei and the likelihood that we will be able to study them in experiment. We have yet to answer the very basic question of "what is the heaviest element?", but we are continuing to learn about the fundamental processes that dictate the answer.

This work was supported in part by the U.S. Department of Energy, Office of Science, Office of Nuclear Physics, under Contract No. DE-AC02-05CH11231(LBNL).

\section{References}

[1] R.-D. Herzberg and P.T. Greenlees, Prog. Part. Nucl. Phys. 61, 674 (2008).

[2] M. Asai, F.P. Heßberger, A. Lopez-Martens, Nucl. Phys. A944, 308 (2015).

[3] S. Hofmann et al., Eur. Phys. J. A10, 5 (2001).

[4] D. Ackermann, Nucl. Phys. A944, 376 (2015).

[5] H.M. David et al., Phys. Rev. Lett. 115, 132502 (2015).

[6] D. Rudolph et al., Phys. Rev. Lett. 111, 112502 (2013).

[7] J.M. Gates et al., Phys. Rev. C 92, 021301(R) (2015).

[8] D. Rudolph, L.G. Sarmiento, U. Forsberg, AIP Conf. Proc. 1681, 030015 (2015).

[9] J. Rissanen et al., Phys. Rev. C 90, 044324 (2014).

[10] U. Forsberg et al., Nucl. Phys. A953, 117 (2016). 
[11] Yu.Ts. Oganessian et al., Phys. Rev. C 87, 014302 (2013).

[12] Yue Shi et al., Phys. Rev. C 90, 014308 (2014).

[13] H. Meldner, Ark. Phys. 36, 593 (1967).

[14] R.R. Chasman et al., Rev. Mod. Phys. 49, 833 (1977).

[15] S. Ćwiok et al., Nucl. Phys. A573, 356 (1994).

[16] A.V. Afansjev et al., Phys. Rev. C 67, 024309 (2003).

[17] M. Bender et al., Nucl. Phys. A723, 354 (2003).

[18] A. Parkhomenko and A. Sobiczewski, Acta. Phys. Pol. B 35, 2447 (2004).

[19] A. Parkhomenko and A. Sobiczewski, Acta. Phys. Pol. B 36, 3115 (2005).

[20] A. Afanasjev and S. Shawaqfeh, Phys. Lett. B 706, 177 (2011).

[21] F. Barranco, R.A. Broglia, G.F. Bertsch, Phys. Rev. Lett. 60, 507 (1988).

[22] F. Barranco, E. Viogezzi, R.A. Broglia, Phys. Rev. C 39, 2101 (1989).

[23] F. Barranco, G.F. Bertsch, R.A. Barranco, E. Vigezzi, Nucl. Phys. A512, 253 (1990).

[24] D.M. Brink and R.A. Broglia, Nuclear Superfluidity (Cambridge University Press, Cambridge, 2005).

[25] F.G. Kondev, G.D. Dracoulis, T. Kibedi, At. Data and Nucl. Data Tables 103-104, 50 (2015), and references therein.

[26] R. Vandenbosch and J.R. Huizenga, Nuclear Fission (Academic Press, 1973).

[27] D.C. Hoffman and M.R. Lane, Radiochimica Acta 70/71, 135 (1995). 